\title{
DYNAMIC OBSTACLE-CROSSING OF A WHEELED ROVER WITH DOUBLE-WISHBONE SUSPENSION
}

\author{
J.C. FAUROUX, B.C. BOUZGARROU
}

Clermont University, French Institute for Advanced Mechanics (IFMA), EA3867, FR TIMS / CNRS 2856, Mechanical Engineering Research Group (LaMI), BP 10448, F63000

\begin{abstract}
In order to evaluate the feasibility of fast terrestrial inspection, this work presents an experimental study of the longitudinal stability of a rapid wheeled rover crossing an obstacle at $10 \mathrm{~m} / \mathrm{s}$. The considered rover uses wheels suspended on double wishbones. Results show that a stability front can be traced, after which tip-over occurs systematically. Impact force measurements and vehicle analytic modeling complete this work and trace future improvement on suspension architecture including additional mobilities.
\end{abstract}

\section{Introduction}

Many mobile all-terrain robots are used for safety and inspection applications but most of them are slow (less than $3 \mathrm{~m} / \mathrm{s}$, Fig. 1). The low speed allow them to use special modes of locomotion such as obstacle climbing modes for military applications (I-Robot Packbot [1]) or industrial inspection (M-Tecks Arthron [2]). Low speed is also suitable for gaming / home use (Meccano Spykee [3]).
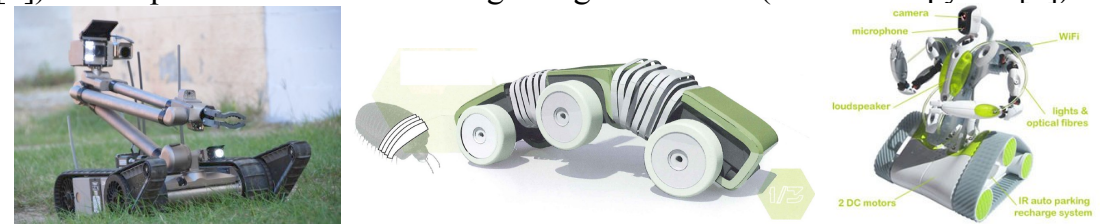

Fig. 1: a) I-Robot Packbot [1]. b) M-Tecks Arthron [2]. c) Meccano Spykee [3].

It appears that many outside applications could benefit from high speed. Inspecting a vast area such as an airport would require less terrestrial robots it they are fast enough. Fast inspection is also more dissuasive. Even if unmanned aerial vehicles and flying drones become popular, they may be dangerous for the human environment (risk of fall) and have limited autonomy because of mass constraints. In this case, terrestrial drones keep their full interest. Fast terrestrial drones could have many other applications in agriculture 
(weeding, seeding), transport of small parts and casualty detection during natural catastrophes.

One major difficulty for fast terrestrial robot in natural environment is to manage obstacle-crossing at high speed. Relatively few works describe what happens when a mobile robot crashes against an obstacle. Some robots can be thrown above obstacles by an operator: they may be equipped by a suspension, such as the 3-spring star-configured suspension of [4] or may be very light with no suspension, such as the DragonRunner [5]. The dynamic crossing of an obstacle is studied in [6] but restricted to low obstacles (e.g. pavement edge).

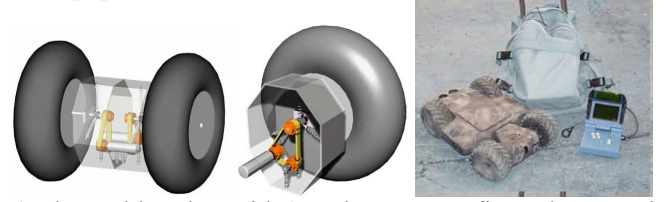

Fig. 2: a) Throwable robot with 3-spring star-configured suspension [4].

b) Ultra-rugged DragonRunner robot without suspension [5].

This work is part of the FAST research project, where the considered fast terrestrial robots use wheel locomotion in all-terrain up to $10 \mathrm{~m} / \mathrm{s}$. This paper will evaluate the longitudinal stability of a classical vehicle (with double wishbone suspensions) crossing a high obstacle at high speed. The violent shock and transient fast phenomena to study led us to use small scale vehicles and to directly experiment before modeling. Section 2 introduces the vehicle and experimental measurements and results. Section 3 develops a simplified analytical model fitting the experimental results.

\section{Experimenting obstacle crossing at high speed}

\subsection{Experimental protocol}

Experiments are performed with an unmodified electric vehicle at scale 1:10 (Fig. 3, E-Maxx \#3903, Traxxas Inc.), weighing 5.16kg for a total length of $518 \mathrm{~mm}$ (track-width $=330 \mathrm{~mm}$, wheelbase $=335 \mathrm{~mm}$ ). With two brushed motors in parallel, the vehicle can reach a maximal speed of $14 \mathrm{~m} / \mathrm{s}$, which exceeds our objective speed of $10 \mathrm{~m} / \mathrm{s}$.
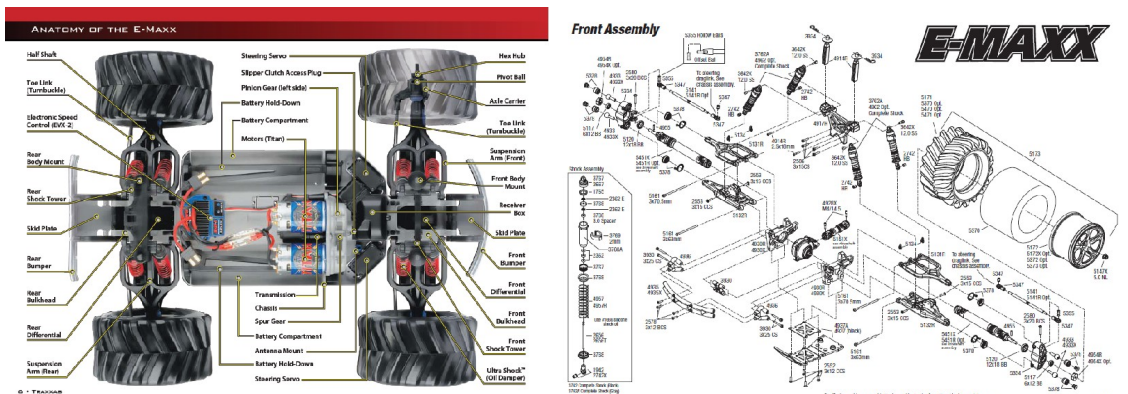

Fig. 3: a) Overview of vehicle architecture. b) View of the front double-wishbone suspension [7]. 
The obstacle is made of a square section steel bar with adjustable height along vertical rails (Fig. 4). The rails are screwed on brackets, that are also screwed on 3-component piezoelectric force sensors (9257B, Kistler Inc.).

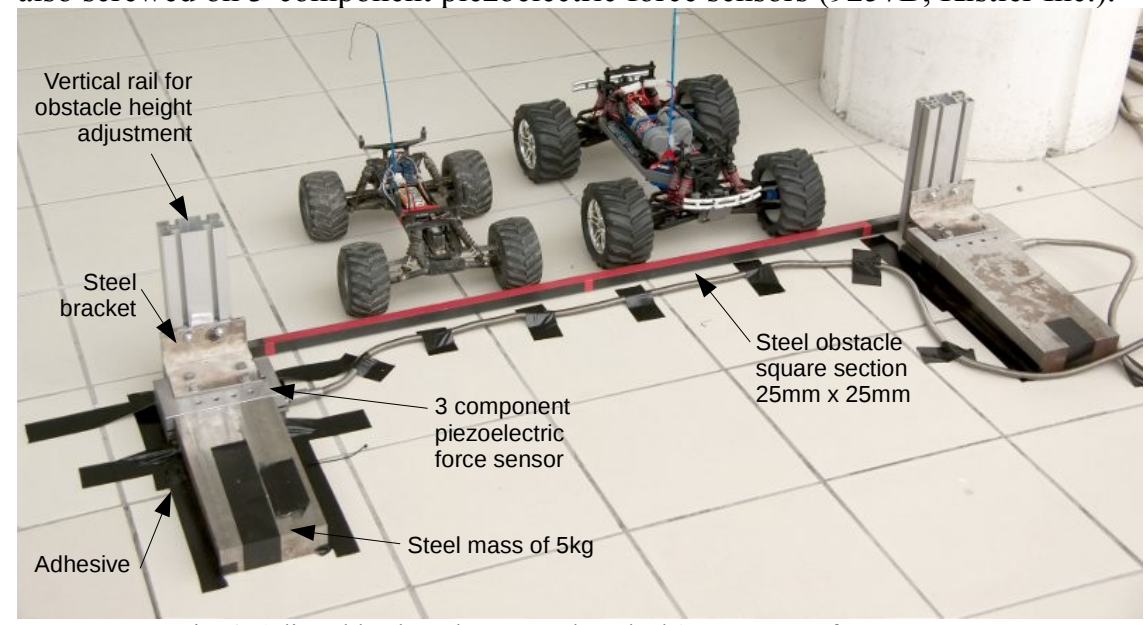

Fig. 4: Adjustable obstacle mounted on dual 3-component force-sensors.

Speed measurements are made by vision with a $30 \mathrm{~Hz}$ camera located above of the impact zone. The vehicle runs on a tiled floor with a periodic pattern of $300 \mathrm{~mm}$. Only the last two images before impact are considered to calculate the instantaneous speed (Fig. 5a).
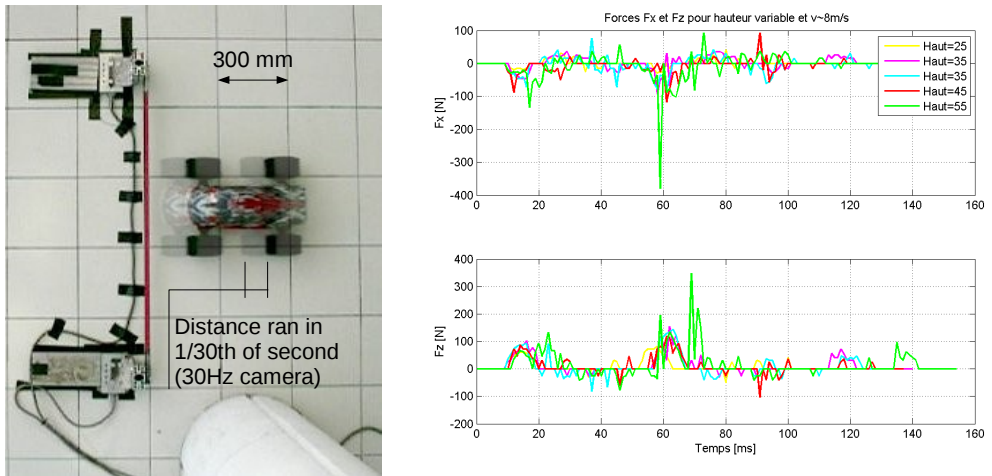

Fig. 5: a) Vision measuring of the instantaneous speed before impact ( $30 \mathrm{~Hz}$ video).

b) Evolution of horizontal $F x$ and vertical $F z$ impact forces according to obstacle height [8]

\subsection{Experimental results}

The experimental force measurements at $1 \mathrm{kHz}$ confirm that the impact force increases with the obstacle-height. For high obstacles, peaks can reach $400 \mathrm{~N}$ and the horizontal force $F x$ has the same order of magnitude as the vertical one $\mathrm{Fz}$ (Fig. 5b). This suggests that vehicles dedicated to fast obstacle crossing should be also designed with a horizontal suspension, as modeled in [9]. 
A total of 77 experiments were performed and summarized in Fig. 6. For high speeds or high obstacles, longitudinal stability is compromised and the vehicle crashes by tip-over (red dots). A stability front that separates experiments with or without tip-over can be traced. It has a decreasing nonlinear shape. Improvement of the stability zone can be expected from the development of a future innovative suspension with 2DDL (vertical+horizontal).

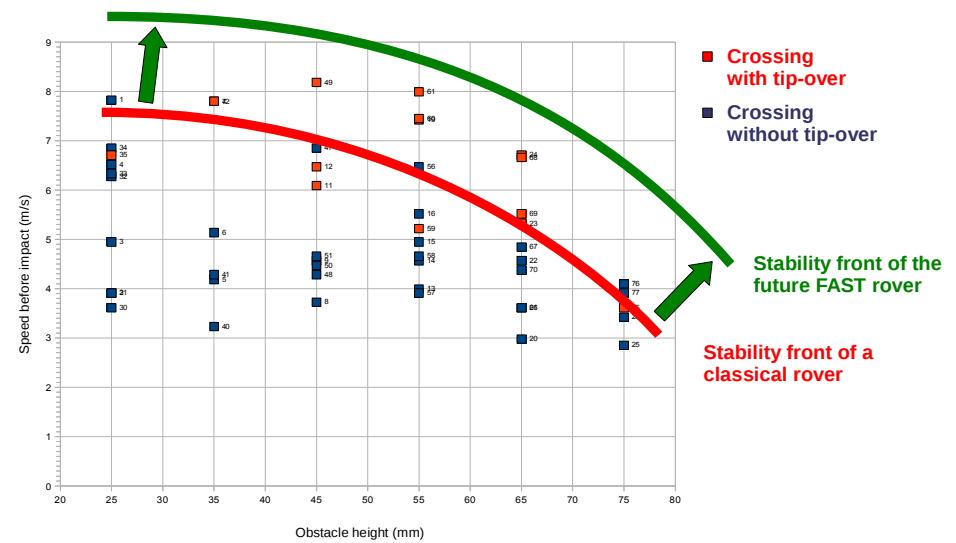

Fig. 6: Summary of all the experimental results with the presence of a stability front.

\section{Simple analytical model}

A simplified model of the vehicle can be set up, based on experimental results and considering only the vertical wheel translations and the pitch angle of the chassis. Therefore, it is proposed to represent the vehicle by a bicycle model with three rigid bodies: two wheels and the chassis. The wheels and the chassis are connected by spring-damper systems. Vehicle suspension mechanisms authorize planar motion of the wheels relatively to the chassis.

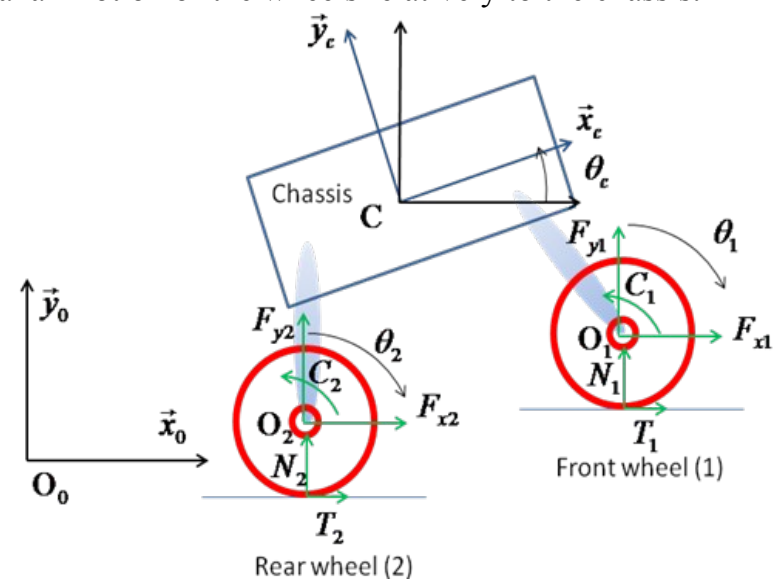

Fig. 7: Parametrization of the vehicle bodies and applied forces. 


\subsection{Parameterization}

We consider a planar motion of the in the $\left(O_{0}, x_{0}, y_{0}\right)$ plane where $R_{0}\left(O_{0}, x_{0}, y_{0}, z_{0}\right)$ is the fixed reference frame (Fig. 7). A frame is attached to each body at its center of mass: $R_{C}\left(O_{C}, x_{C}, y_{C}\right)$ for the chassis, $R_{1}\left(O_{1}, x_{1}, y_{1}\right)$ for the front wheel and $R_{2}\left(O_{2}, x_{2}, y_{2}\right)$ for the rear wheel.

The positions of $R_{C}$ relatively to $R_{0}$, projected in $R_{0}$, is given by $\overrightarrow{O_{0} \vec{O}_{C}}=X_{C} \vec{x}_{0}+Y_{C} \vec{y}_{0}$ and its orientation is given by the rotation angle $\theta_{C}=\Varangle\left(\vec{x}_{0}, \vec{x}_{C}\right)=\Varangle\left(\vec{y}_{0}, \vec{y}_{C}\right)$. These parameters are grouped in the position vector $\left(X_{C}, Y_{C}, \theta_{C}\right)$. In the same way, the positions and orientations relatively to $R_{0}$ of frames $R_{1}$ and $R_{2}$ attached to the wheels are given by $\left(X_{1}, Y_{1}, \theta_{l}\right)$ and $\left(X_{2}, Y_{2}, \theta_{2}\right)$.

\subsection{Equations of motion}

By isolating separately vehicle solids, three dynamic equations can be written for each one (Newton-Euler equations). The chassis is submitted to wheel forces and torques as well as gravity (Fig. 7). The following equations can be written:

$$
\begin{aligned}
& m_{c} \ddot{X}_{c}=F_{x 1}+F_{x 2} \\
& m_{c} \ddot{Y}_{c}=F_{y 1}+F_{y 2}-m_{c} g \\
& I_{c z} \ddot{\theta}_{c}=M_{1}+M_{2}-C_{1}-C_{2}
\end{aligned}
$$

where $F_{x i}$ and $F_{x i}(i=1,2)$ are the joint forces applied by the wheel $(i)$ on the chassis. These forces depend on suspension loading. $C_{1}$ and $C_{2}$ are the torques applied by the wheel motors. $M_{1}$ and $M_{2}$ denote the moments of the wheel force at the chassis center of mass point $C . m_{C}$ and $I_{C Z}$ are respectively the mass and the moment of inertia about $z_{C}$ of the chassis.

The following equations can be written for each wheel:

$$
\begin{aligned}
& m_{i} \ddot{X}_{i}=T_{i}+R_{x i}-F_{x i} \\
& m_{i} \ddot{Y}_{i}=N_{i}+R_{y i}-F_{y i}-m_{i} g \\
& I_{z i} \ddot{\theta}_{i}=C_{i}+r T_{i}
\end{aligned}
$$

where $N_{i}$ and $T_{i}$ are respectively the normal and the tangential wheel - ground contact forces (Fig. 7). $R_{x i}$ and $R_{y i}$ are the additional obstacle impact forces. $m_{i}$ and $I_{z i}$ are respectively the mass and the moment of inertia of the wheel $(i)$.

\subsection{Wheel ground contact}

In vehicle dynamics, the wheel - ground contact modeling is a critical issue since contact forces are dependent on the vehicle motion that they generate. The normal contact forces are given by:

$$
N_{i}=\left\{\begin{array}{cc}
k\left(r-Y_{i}\right)-c \dot{Y}_{i} & \left(Y_{i} \leq r\right) \\
0 & \left(Y_{i}>r\right)
\end{array}\right.
$$

where $k$ and $c$ are respectively the stiffness and damping of the wheel - ground unilateral contact. The tangential contact force is modeled by equation (4). 
6

$$
T_{i}=N_{i} \min \left(g_{r} \mu-\left(1-g_{r}\right) \frac{C_{i}}{r N_{i}}, g_{r} \mu\right)
$$

This formula takes into account the relative slipping $g_{r}$, given by equation 5 , and uses one friction parameter $\mu$, which can be identified experimentally. Contrarily to classic Pacejka like formulae, this formula can be used even in static equilibrium and allows rolling without slipping condition.

$$
g_{r}=\frac{-r \dot{\theta}_{i}-\dot{X}_{i}}{\max \left(\left|r \dot{\theta}_{i}\right|,\left|\dot{X}_{i}\right|\right)}
$$

Finally, the impact force, applied by the obstacle to the wheel is modeled by the action of a linear spring-damper (Fig. 8). This force is proportional to the wheel - obstacle penetration $e$ and oriented from the contact point to the wheel center.

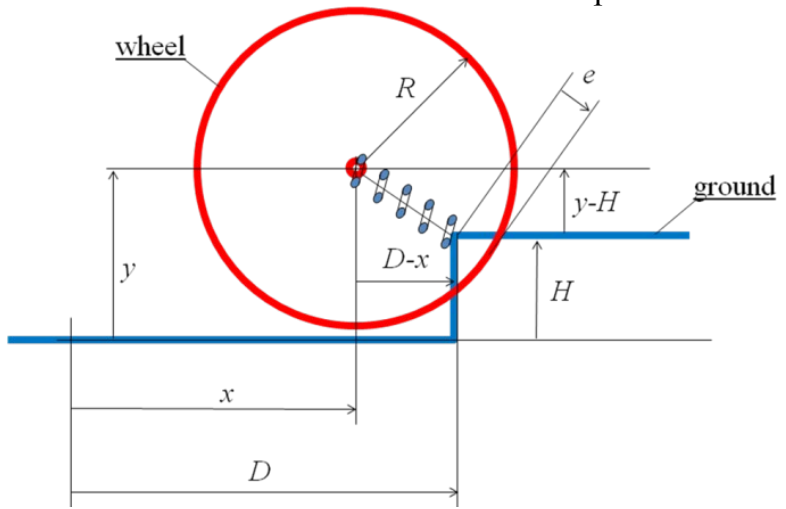

Fig. 8: Impact force modeling.

The spring-damper model was chosen after analyzing a wheel impact with a high speed camera at $10000 \mathrm{~Hz}$ (Fig. 9). Our reduced car is equipped with rubber tires wrapping a foam layer. Tires have no pressure (decompression holes in the rim). The video clearly showed two steps: in a first step, tire compression; in a second step, hard shock on the rim of the wheel (images 3-4 from the left). In this model, only step 1 was represented. Future work could also include step 2.

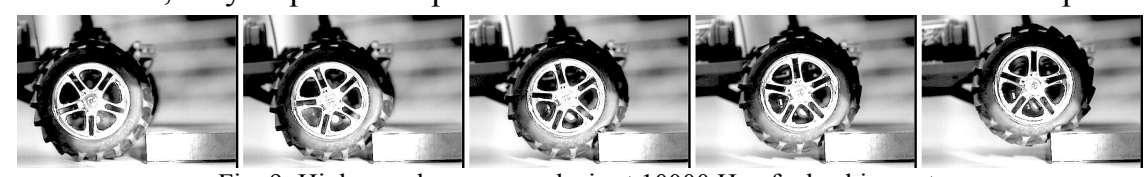

Fig. 9: High speed camera analysis at $10000 \mathrm{~Hz}$ of wheel impact.

\subsection{Simulation results}

By using the elaborated model, crossing operations can be simulated for several values of impact velocity and obstacle height. As expected in reality, a tip-over failure is obtained for relatively low speed and high obstacle or high speed and low obstacle. These simulations are quite realistic and allow analyzing crossing 
conditions. They also enable design optimization by tuning suspension stiffness and damping. A simulated motion is represented in Fig. 10. In this case, the impact speed is $5 \mathrm{~m} / \mathrm{s}$ and the obstacle height is $5 \mathrm{~cm}(2 / 3$ of the wheel radius). The vehicle succeeded to cross the obstacle but the trajectory contains ballistic phases and two bounces.

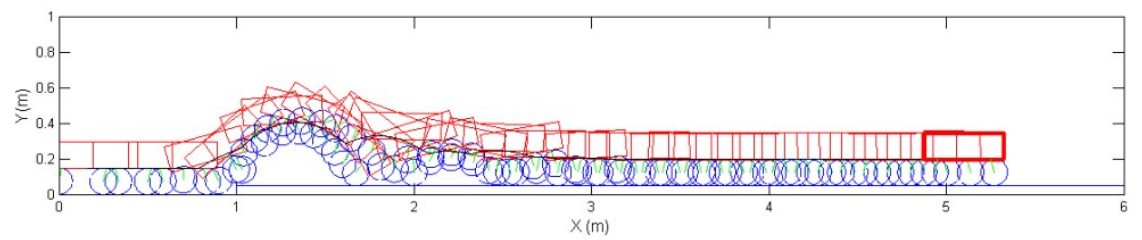

Fig. 10: Trajectory simulation.

In order to analyze the obstacle crossing capacity of the vehicle, a numerical design of experiment (DoE) was performed with two variables: the impact speed and the obstacle height. With only two variables, a full factorial DoE was achievable with ten levels for each variable. Fig. 11 shows the result of the DoE on one hundred of virtual crossings. Each simulation can lead to four possible situations, combination of two factors : obstacle crossing (yes or no) and stability (tip-over or not).

We can identify a successful and stable crossing zone under the blue bold circle points. A precise boundary of this zone can be obtained by a finer meshing of the crossing parameters around these points. It can be noticed the existence of some isolated zones or points which can't be exploited safely in a real application.
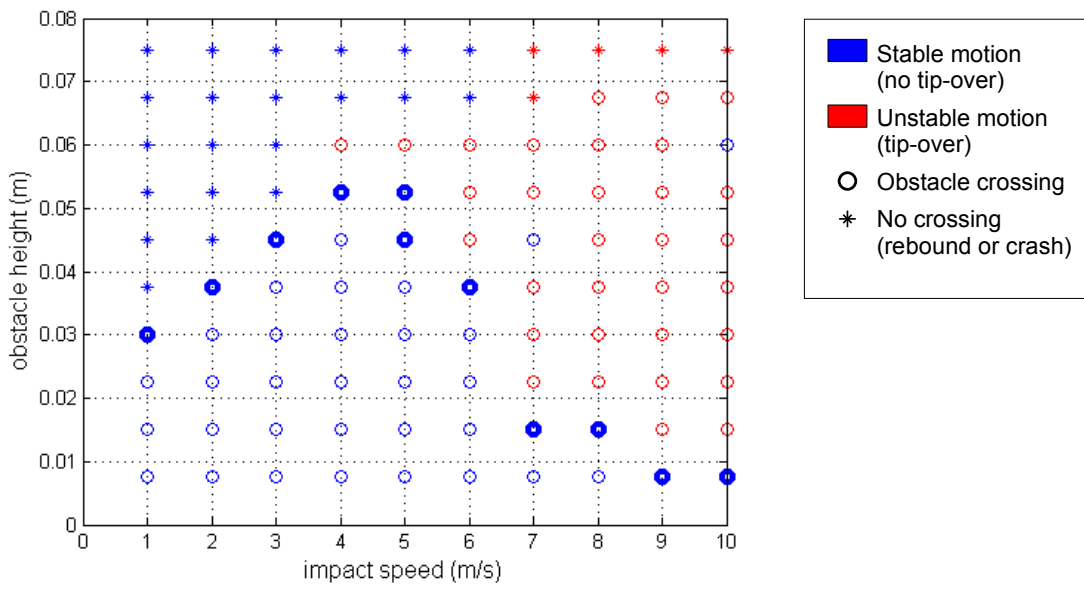

Fig. 11: Numerical characterization of crossing capacity and stability.

\section{Conclusion}

In this work, 77 experiments of dynamic obstacle-crossing were performed with an electric vehicle at scale $1 / 10$. It allowed showing that there exists a stability 
limit after which the vehicle crashes systematically by tipping over. This limit can be expressed as a non-linear relation between the vehicle speed and the obstacle height. The vehicle can cross low obstacles at high speed or high obstacles at low speed. This means that the speed limit for keeping the vehicle controllable can be directly inferred from sensing obstacle height.

This behaviour was corroborated by an analytical model based on a bicycle model and simple equations of contact forces. The model was tested in a design of experiment containing one hundred virtual crossings.

Experimental measurement of impact forces showed that, for steep obstacles that are typical of all-terrain use, the horizontal component of the contact force can even exceed the vertical component. This traces a major perspective of improvement for dynamic obstacle-crossing: creating innovative suspensions that are capable to also absorb horizontal shocks.

Future work include developing a new kinematics of suspension to absorb horizontal shocks; refining the impact model by adding a non constant stiffness; finding the analytical expression of the frontier between stable and unstable behaviour; and last but not least: choosing a control strategy for optimal obstacle crossing.

\section{Acknowledgments}

This work is supported by the French National Research Agency and is included into the ANR FAST project. The authors wish to thank Richard COUSTURIER for help in experiments, Miguel CANO for piloting and Jamil DAKHLALLAH for post-processing.

\section{References}

1. iRobot Inc. 510 Packbot robot specifications. http://www.irobot.com

2. M-Tecks EAC Inc. Arthron-R robot. http://www.m-teckseac.com

3. Meccano Inc. Spykee robot. http://www.meccano.com/models/spykee

4. D. O'Halloran, A. Wolf and H. Choset. Design of a high-impact survivable robot. Mechanism and Machine Theory. 40, 1345-1366 (2005).

5. H. Schempf, W. Crowley, C. Gasior and D. Moreau. Ultra-rugged SoldierRobot for Urban Conflict Missions. Unmanned Systems 2003 Conference AUVSI 30th Annual Symposium and Exhibition, Baltimore, MD, July 15-17 (2003). http://www.rec.ri.cmu.edu/projects/dragonrunner/tech

6. H.B. Pacejka, Tyre And Vehicle Dynamics, Butterworth-Heinemann, 2005.

7. Traxxas Inc. E-Maxx\#3903, 1/10 scale, 4WD electric Monster truck, Reference manual and Part list. http://traxxas.com/products/models/electric/3903emaxx-downloads

8. J. Dakhlallah, Design of mechatronics systems for innovative suspensions dedicated to high speed off-road mobile robots, Post-doctoral report (2010).

9. J.C. Fauroux, J. Dakhlallah, B.C. Bouzgarrou. A New Concept of FAST Mobile Rover with Improved Stability on Rough Terrain. Proc. of HUDEM'2010, 8th IARP Workshop on Robotics and Mechanical assistance in Humanitarian De-mining, Sousse, Tunisia. 16 p, 10-12 May (2010). 